\title{
Fractional approach for model of network access control using efficient method
}

\author{
Esin ILLHAN* \\ Faculty of Engineering and Architecture, Kirsehir Ahi Evran University, Kirsehir, 40500, Turkey \\ Geliş Tarihi (Received Date): 15.09.2020 \\ Kabul Tarihi (Accepted Date): 29.12.2020
}

\begin{abstract}
In this paper, we find the solution for the system of nonlinear ordinary differential equations having fractional-order arising in network access control using fractional natural decomposition method (FNDM). The consider a model which consists of a system of five nonlinear ordinary differential equations (NODEs), which illustrate the sensor networks are interesting essentials for malicious outbreaks that attack the network with the intention of reducing the integrity, availability and confidentiality. Further, we captured the nature of FNDM results for different value of fractional order in terms of the plots. The considered scheme highly effective and structured while examining nonlinear models and which can be observed and confirm from the obtained results. Further, the conspiracies cited in plots confirm the hired fractional operator and algorithm can help to exemplify the more fascinating properties of the nonlinear system associated real-world problems.
\end{abstract}

Keywords: Caputo derivative, network access control, mathematical model, fractional natural decomposition method.

\section{Etkili yöntem kullanan ağ erişim kontrolü modeli için kesirli yaklaşım}

$\ddot{\mathbf{O} z}$

Bu makalede, kesirli doğal ayrıştırma yöntemi (FNDM) kullanarak ă erişim kontrolünde ortaya çıkan kesirli mertebeye sahip doğrusal olmayan adi diferansiyel denklemler sistemi için çözüm buluyoruz. Sensör ağlarını göstermek için kullanılan ve beş doğrusal olmayan adi diferansiyel denklem (NODE) sisteminden oluşan bu model, bütünlüğü, kullanılabilirliği ve gizliliği azaltmak amacıyla ăga saldıranlar için ilginç

\footnotetext{
* Esin İLHAN, eilhan@ahievran.edu.tr, https://orcid.org/0000-0002-0839-0942
} 
temel özelliklere sahiptir. Ayrıca, grafikler açısından farklı kesirli parametre değerleri için FNDM çözümlerinin grafiklerini de çizdik. Dikkate alınan method, doğrusal olmayan modelleri incelerken oldukça etkili ve yapılandırılmıştır ve elde edilen sonuçlardan gözlemlenebilir ve doğrulanabilirdir. Üstelik, grafiklerden elde edilen verilerden, kesirli operatörlerin, gerçek dünya problemlerinin kaydadeğer özelliklerini ortaya çıkarmak için oldukça yardımedebilir olduğu doğrulanır.

Anahtar kelimeler: Caputo türevi, ăg erişim kontrol model, matematiksel model, kesirli natural ayrıştırma yöntemi.

\section{Introduction}

The analysis of nature and its constituents with the aid of mathematical models have attracted researchers due to their significant contributions towards the development of essentials. The mathematical models can provide exact and effective predictions of the phenomena in a short time without causing any loss to nature and its constituents. In this connection, differential calculus gets more and more notices and it is the only instrument which can perfectly exemplify the mechanism with the rate of change. There have numerous procedures to employ this tool to nurture the phenomena raised in daily life. Recently mathematicians and scientists are illustrated and proved that the concept of fractional calculus (FC) is more appropriated as related to the classical concept. Moreover, it can capture more essential and interesting consequences as compared to traditional calculus. Even though, there are plenty of open questions are raised with FC, due to the revolution of technology within the frame of computer many scholars show tremendous interest in this concept and derived numerous stimulating results. There are diverse notions exemplified by many mathematicians to enrich the concept and lead the foundations for FC [1-33].

On other hand, Wireless Sensor Networks (WSN) attracted the attention of many researchers due to its gigantic technology in the present scenario. Without engineered position or predetermined in untrusted terrains, these networks permit the random positioning of sensor-nodes. This significant technology is miniaturized; however low powered battery devices that integrate minimal sensing, radio transmission capabilities, computation and processing [34]. Due to adaptable and dynamic architectures and also above-mentioned virtues, it has been widely used in the military, industries, healthcare's, and homes. Particularly, in order to monitoring targeting, evaluation of battle damage, reconnaissance, battlefield surveillance, equipment measurement and other pivotal essence are examined by the help of WSN. In the environment and homes, WSN is considering in order detecting the flood and fire, it helps to map the biocomplexity and mainly consider agriculture precision. Recently, it has been considered as an essential instrument in the commercial purpose like drug and patients administration telemonitoring and others [35].

In the present investigation, we consider a system of five equations describing the nature of the model of wireless sensor network which aid to investigate transmission range, distribution density, and sensor field/area. Without human intervention or physical contact, in sensor networks recent innovation-based advances designate the malevolent attacker can operate numerous innards of sensor nodes to outspread malicious [36]. Moreover, the communication happens in the medium of open-air [37] 
the WSN an easy target to external attacks due to and its distribution in unfriendly, untrusted, unguarded terrain. Further, the open challenges exploit due to these attackers to WSN including, uncertainty, communication range, computational power and finite bandwidth, and others [34, 35].

In order to perfectly characterize the malicious code banquet with time in a sensor network, authors in [38] suggest the NCA-Susceptible-Exposed-InfectiousRemediated-Vaccinated (NAC-SEIRV) epidemic model with the inclusion of transmission range $\left(r^{2}\right)$ and distribution density $(\sigma)$. Initially, the sensor nodes transient via the pre-screening process and sent to the remediated compartment after the infectious immigrant nodes are treated and isolated. Sensor nodes enter the susceptible sensor population when they are free of any malicious code infection. To the outbreak of subsequent malicious code infections, Immunization of the predisposed sensor nodes offers supplementary immunization competence.

For the compartment of NCA and its dynamics is described with the aid of the following relation

$\frac{d Q(t)}{d t}=\lambda-\mathcal{Q}(\omega+\eta+\rho+d)$

where $\lambda$ is inclusion rate of nodes, $\omega$ is the transmission rate to remediated class from quarantine class, $\eta$ is the rate of death infected immigrant nodes, $\rho$ is the transmission rate to susceptible class from quarantined. With the aid of convolution integral, the solution for the above equation with the initial population $\mathcal{Q}_{0}$, as follows

$\mathcal{Q}(t)=e^{-(\omega+\eta+\rho+d) t} \mathcal{Q}_{0}+\frac{\lambda}{\omega+\eta+\rho+d}\left(1-e^{-(\omega+\eta+\rho+d) t}\right)$.

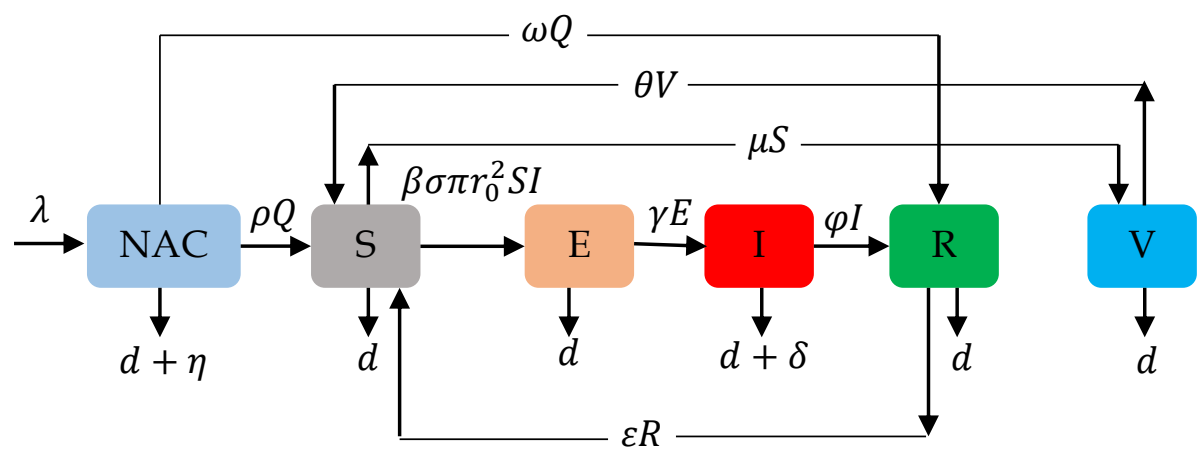

Figure 1. Schematic diagram of NAC-SEIRV model [25].

In Figure 1, the schematic diagram is presented and the dynamical transfer of the population as of assumptions and with the help of this a system of NODEs are generated with susceptible $(S)$, exposed $(E)$, infectious $(I)$, recovered $(R)$ and vaccinated $(V)$, and which is of the form of [38]

$$
\begin{aligned}
& \frac{d S(t)}{d t}=\rho Q(t)+\theta V(t)+\varepsilon R(t)-\left(\beta \sigma \pi r_{0}^{2} I(t)+\mu+d\right) S(t), \\
& \frac{d E(t)}{d t}=\beta \sigma \pi r_{0}^{2} S(t) I(t)-(\gamma+d) E(t), \\
& \frac{d I(t)}{d t}=\gamma E(t)-(\varphi+\delta+d) I(t), \\
& \frac{d R(t)}{d t}=\omega Q(t)+\varphi I(t)-(\varepsilon+d) R(t),
\end{aligned}
$$


$\frac{d V(t)}{d t}=\mu S(t)-(\theta+d) V(t)$

Here, $\rho$ and $\varepsilon$ respectively signifies transmission rate from quarantined to susceptible and remediated to susceptible classes, $\theta$ indicate transmission rate from vaccinating to susceptible, $\beta$ denotes the rate of infectivity contact, for the susceptible nodes, $\sigma$ is the coefficient rate of vaccinating, due to failure of software or hardware, rate of death or mortality nodes is symbolised by $d, \gamma$ represents the transmission rate from exposure to infectious, $\varphi$ and $\omega$ are respectively denote the transmission rate from infectious to remediated and quarantine to remediated, $\delta$ represents the rate of crashing due to attack of worms and $\eta$ rate of death of the infected immigrant nodes.

The fundamental notions and associated rules of FC are extensively hired by many scholars to exemplify their viewpoints about the numerous classes of nonlinear phenomena. Specifically, the solitons solutions for optic problems, the mechanisms and processes exist in nanotechnology, to study the behaviour of the human diseases with mathematical models, to examine the behaviour of systems related to chaotic behaviour, and many other activities and phenomena. By incorporating this concept to any system, we get more degree of freedom to examine the corresponding phenomena. However, day by day new operators with respect to fractional calculus have been proposed by many researchers to overcome formerly acknowledged limitations and drawbacks.

In the present framework, we consider the system (1) with the novel fractional operator of the form

$$
\begin{aligned}
& D_{t}^{\alpha} S(t)=\rho Q(t)+\theta V(t)+\varepsilon R(t)-\left(\beta \sigma \pi r_{0}^{2} I(t)+\mu+d\right) S(t), \\
& D_{t}^{\alpha} E(t)=\beta \sigma \pi r_{0}^{2} S(t) I(t)-(\gamma+d) E(t), \\
& D_{t}^{\alpha} I(t)=\gamma E(t)-(\varphi+\delta+d) I(t), \\
& D_{t}^{\alpha} R(t)=\omega Q(t)+\varphi I(t)-(\varepsilon+d) R(t), \\
& D_{t}^{\alpha} V(t)=\mu S(t)-(\theta+d) V(t),
\end{aligned}
$$

where $\alpha$ is fractional order of the system.

The study of mathematical models, specifically differential systems is the most attracted area of research and numerous new ideas, results and notions with interesting significances are derived. But, humankind is unrest, every day coming with new ideas and novel tools to investigate essential properties. From lost twenty years, numbers of new solution procedures are proposed by many senior scholars to find the solution for the systems. In this regard, Adomian decomposition technique [39] is also one of them and it has been extensively employed by many others to investigate diverse family and classes of problems without perturbation and conversion for PDEs to ODEs and nonlinear to linear. However, some authors nurtured as unions with familiar transform schemes, this method becomes more powerful, it consumes less time to evaluate the terms and increase the reliability of the method. The considered method is a merge of the above-cited method with natural transform [40] suggested by Rawashdeh and Maitama [41, 42]. Later, it hired by many authors to find and analyse the corresponding nature of the solution for many real-world problems [43-47]. The effort of many scientists and mathematicians in the area of computer technology and its security is magnetized many authors. The projected model and its similar development are analysed by many scholars [48-55]. 


\section{Preliminaries}

In this segment, we recall some basic and fundamentals of FC.

Definition 1. In the fractional Riemann-Liouville sense, the integral of a function $f(t) \in C_{\delta}(\delta \geq-1)$ is presented as follows [1]

$J^{\alpha} f(t)=\frac{1}{\Gamma(\mu)} \int_{0}^{t}(t-\vartheta)^{\mu-1} f(\vartheta) d \vartheta$

Definition 2. The Caputo fractional derivative of $f \in C_{-1}^{n}$ is presented as [2]

$D_{t}^{\alpha} f(t)= \begin{cases}\frac{d^{n} f(t)}{d t^{n}}, & \alpha=n \in \mathbb{N}, \\ \frac{1}{\Gamma(n-\alpha)} \int_{0}^{t}(t-\vartheta)^{n-\alpha-1} f^{(n)}(\vartheta) d \vartheta, & n-1<\alpha<n, n \in \mathbb{N} .\end{cases}$

Definition 3. The Mittag-Leffler type function with one-parameter is defined [56] as follows

$E_{\alpha}(z)=\sum_{k=0}^{\infty} \frac{z^{k}}{\Gamma(\alpha k+1)}, \alpha>0, z \in \mathbb{C}$.

Definition 4. The natural transform (NT) of $f(t)$ is symbolized by $\mathbb{N}[f(t)]$ for $t \in \mathbb{R}$ and presented with the NT variables $s$ and $\omega$ by [40]

$\mathbb{N}[f(t)]=R(s, \omega)=\int_{-\infty}^{\infty} e^{-s t} f(\omega t) d t ; \quad s, \omega \in(-\infty, \infty)$.

Now, we define the NT with Heaviside function $H(t)$ as

$\mathbb{N}[f(t) H(t)]=\mathbb{N}^{+}[f(t)]=R^{+}(s, \omega)=\int_{0}^{\infty} e^{-s t} f(\omega t) d t ; s, \omega \in(0, \infty)$.

Theorem 1 [40]: The NT $R_{\alpha}(s, \omega)$ of the fractional derivative of $f(t)$ RiemannLiouville sense is symbolized by $D^{\alpha} f(t)$ and defined as

$\mathbb{N}^{+}\left[D^{\alpha} f(t)\right]=R_{\alpha}(s, \omega)=\frac{s^{\alpha}}{\omega^{\alpha}} R(s, \omega)-\sum_{k=0}^{n-1} \frac{s^{k}}{\omega^{\alpha-k}}\left[D^{\alpha-k-1} f(t)\right]_{t=0}$,

where $R(s, \omega)$ is NT of $f(t), \alpha$ is the order and $n$ be any positive integer. Further, $n-$ $1 \leq \alpha<n$.

Theorem 2 [57]: The natural transform $R_{\alpha}(s, \omega)$ of the arbitrary derivative in Caputo sense of $f(t)$ is symbolize by ${ }^{c} D^{\alpha} f(t)$ and defined as

$\mathbb{N}^{+}\left[{ }^{c} D^{\alpha} f(t)\right]=R_{\alpha}^{c}(s, \omega)=\frac{s^{\alpha}}{\omega^{\alpha}} R(s, \omega)-\sum_{k=0}^{n-1} \frac{s^{\alpha-(k+1)}}{\omega^{\alpha-k}}\left[D^{k} f(t)\right]_{t=0}$.

\section{Fundamental solution procedure of the projected method}


Here, we consider coupled system in order to illustrate the basic solution procedure of the considered algorithm with initial conditions

$D_{t}^{\alpha} u(x, t)+R u(x, t)+F u(x, t)=h_{1}(x, t)$,

$D_{t}^{\alpha} v(x, t)+R v(x, t)+F v(x, t)=h_{2}(x, t)$,

and

$u(x, 0)=g_{1}(x)$

$v(x, 0)=g_{2}(x)$

where $D^{\alpha} u(x, t)$ and $D^{\alpha} v(x, t)$ respectively signifies the fractional Caputo derivatives of $u(x, t)$ and $v(x, t), h_{1}(x, t)$ and $h_{2}(x, t)$ are the source terms. $F$ and $R$ respectively the nonlinear and linear differential operator. On applying $N T$ and with the help of Theorem 2, then Eq. (9) gives

$$
\begin{aligned}
U(x, s, \omega) & =\frac{u^{\alpha}}{s^{\alpha}} \sum_{k=0}^{n-1} \frac{s^{\alpha-(k+1)}}{\omega^{\alpha-k}}\left[D^{k} u(x, t)\right]_{t=0}+\frac{\omega^{\alpha}}{s^{\alpha}} \mathbb{N}^{+}\left[h_{1}(x, t)\right] \\
& -\frac{\omega^{\alpha}}{s^{\alpha}} \mathbb{N}^{+}[R v(x,, t)+F u(x, t)], \\
V(x, s, \omega) & =\frac{v^{\alpha}}{s^{\alpha}} \sum_{k=0}^{n-1} \frac{s^{\alpha-(k+1)}}{\omega^{\alpha-k}}\left[D^{k} v(x, t)\right]_{t=0}+\frac{\omega^{\alpha}}{s^{\alpha}} \mathbb{N}^{+}\left[h_{2}(x, t)\right] \\
& -\frac{\omega^{\alpha}}{s^{\alpha}} \mathbb{N}^{+}[R u(x, t)+F v(x, t)] .
\end{aligned}
$$

On employing inverse $N T$ on Eq. (11) to get

$$
\begin{aligned}
& u(x, t)=G(x, t)-\mathbb{N}^{-1}\left[\frac{\omega^{\alpha}}{s^{\alpha}} \mathbb{N}^{+}[R v(x, t)+F u(x, t)]\right], \\
& v(x, t)=H(x, t)-\mathbb{N}^{-1}\left[\frac{\omega^{\alpha}}{s^{\alpha}} \mathbb{N}^{+}[R u(x, t)+F v(x, t)]\right] .
\end{aligned}
$$

From given initial conditions, non-homogeneous terms, $G(x, t)$ and $H(x, t)$ exists. The infinite series solution is presented as

$$
\begin{array}{ll}
u(x, t)=\sum_{n=0}^{\infty} u_{n}(x, t), & F u(x, t)=\sum_{n=0}^{\infty} A_{n}, \\
v(x, t)=\sum_{n=0}^{\infty} v_{n}(x, t), & F v(x, t)=\sum_{n=0}^{\infty} B_{n},
\end{array}
$$

where $A_{n}$ and $B_{n}$ are indicating the nonlinear terms of $F u(x, t)$ and $F v(x, t)$, respectively. By using the Eqs. (12) and (13), we have

$$
\begin{aligned}
& \sum_{n=0}^{\infty} u_{n}(x, t)=G(x, t)-\mathbb{N}^{-1}\left[\frac{\omega^{\alpha}}{s^{\alpha}} \mathbb{N}^{+}\left[R \sum_{n=0}^{\infty} v_{n}(x, t)\right]+\sum_{n=0}^{\infty} A_{n}\right], \\
& \sum_{n=0}^{\infty} v_{n}(x, t)=H(x, t)-\mathbb{N}^{-1}\left[\frac{\omega^{\alpha}}{s^{\alpha}} \mathbb{N}^{+}\left[R \sum_{n=0}^{\infty} u_{n}(x, t)\right]+\sum_{n=0}^{\infty} B_{n}\right] .
\end{aligned}
$$

By comparing both sides of Eq. (14), we obtain 


$$
\begin{aligned}
& u_{0}(x, t)=G(x, t), \\
& u_{1}(x, t)=-\mathbb{N}^{-1}\left[\frac{\omega^{\alpha}}{s^{\alpha}} \mathbb{N}^{+}\left[R v_{0}(x, t)\right]+A_{0}\right], \\
& u_{2}(x, t)=-\mathbb{N}^{-1}\left[\frac{\omega^{\alpha}}{s^{\alpha}} \mathbb{N}^{+}\left[R v_{1}(x, t)\right]+A_{1}\right], \\
& v_{0}(x, t)=H(x, t), \\
& v_{1}(x, t)=-\mathbb{N}^{-1}\left[\frac{\omega^{\alpha}}{s^{\alpha}} \mathbb{N}^{+}\left[R u_{0}(x, t)\right]+B_{0}\right], \\
& v_{2}(x, t)=-\mathbb{N}^{-1}\left[\frac{\omega^{\alpha}}{s^{\alpha}} \mathbb{N}^{+}\left[R u_{1}(x, t)\right]+B_{1}\right],
\end{aligned}
$$

Similarly, we can obtain the recursive relation in general form for $n \geq 1$ and defined as

$u_{n+1}(x, t)=-\mathbb{N}^{-1}\left[\frac{\omega^{\alpha}}{s^{\alpha}} \mathbb{N}^{+}\left[R v_{n}(x, t)\right]+A_{n}\right]$,

$v_{n+1}(x, t)=-\mathbb{N}^{-1}\left[\frac{\omega^{\alpha}}{s^{\alpha}} \mathbb{N}^{+}\left[R u_{n}(x, t)\right]+B_{n}\right]$.

Lastly, the approximate solutions are defined as follows

$u(x, t)=\sum_{n=0}^{\infty} u_{n}(x, t), \quad v(x, t)=\sum_{n=0}^{\infty} v_{n}(x, t)$.

\section{FNDM solution for the projected system}

Here, we consider the fractional-order system of equations presented in Eq. (2) to find the solution for using projected solution procedure. Then

$$
\begin{cases}D_{t}^{\alpha} S(t)=\rho Q(t)+\theta V(t)+\varepsilon R(t)-\left(\beta \sigma \pi r_{0}^{2} I(t)+\mu+d\right) S(t), & \\ D_{t}^{\alpha} E(t)=\beta \sigma \pi r_{0}^{2} S(t) I(t)-(\gamma+d) E(t), & 0<\alpha \leq 1, \\ D_{t}^{\alpha} I(t)=\gamma E(t)-(\varphi+\delta+d) I(t), & \\ D_{t}^{\alpha} R(t)=\omega Q(t)+\varphi I(t)-(\varepsilon+d) R(t), & \\ D_{t}^{\alpha} V(t)=\mu S(t)-(\theta+d) V(t), & \end{cases}
$$

associated with initial conditions

$S(0)=S_{0}(t), E(0)=E_{0}(t), I(0)=I_{0}(t), R(0)=R_{0}(t), V(0)=V_{0}(t)$

By the assist of NTon Eq. (16), one can get

$$
\begin{aligned}
& \mathbb{N}^{+}\left[D_{t}^{\alpha} S(t)\right]=\mathbb{N}^{+}\left[\rho Q(t)+\theta V(t)+\varepsilon R(t)-\left(\beta \sigma \pi r_{0}^{2} I(t)+\mu+d\right) S(t)\right], \\
& \mathbb{N}^{+}\left[D_{t}^{\alpha} E(t)\right]=\mathbb{N}^{+}\left[\beta \sigma \pi r_{0}^{2} S(t) I(t)-(\gamma+d) E(t)\right] \\
& \mathbb{N}^{+}\left[D_{t}^{\alpha} I(t)\right]=\mathbb{N}^{+}[\gamma E(t)-(\varphi+\delta+d) I(t)] \\
& \mathbb{N}^{+}\left[D_{t}^{\alpha} R(t)\right]=\mathbb{N}^{+}[\omega Q(t)+\varphi I(t)-(\varepsilon+d) R(t),] \\
& \mathbb{N}^{+}\left[D_{t}^{\alpha} V(t)\right]=\mathbb{N}^{+}[\mu S(t)-(\theta+d) V(t)]
\end{aligned}
$$


The non-linear operator is define as

$$
\begin{aligned}
& \frac{s^{\alpha}}{W^{\alpha}} \mathbb{N}^{+}[S(t)]-\sum_{k=0}^{n-1} \frac{s^{\alpha-(k+1)}}{\omega^{\alpha-k}}\left[D^{k} S\right]_{t=0} \\
& =\mathbb{N}^{+}\left[\rho Q(t)+\theta V(t)+\varepsilon R(t)-\left(\beta \sigma \pi r_{0}^{2} I(t)+\mu+d\right) S(t)\right], \\
& \frac{s^{\alpha}}{W^{\alpha}} \mathbb{N}^{+}[E(t)]-\sum_{k=0}^{n-1} \frac{S^{\alpha-(k+1)}}{\omega^{\alpha-k}}\left[D^{k} E\right]_{t=0}=\mathbb{N}^{+}\left[\rho \beta \sigma \pi r_{0}^{2} S(t) I(t)-(\gamma+d) E(t)\right], \\
& \frac{s^{\alpha}}{W^{\alpha}} \mathbb{N}^{+}[I(t)]-\sum_{k=0}^{n-1} \frac{s^{\alpha-(k+1)}}{\omega^{\alpha-k}}\left[D^{k} I\right]_{t=0}=\mathbb{N}^{+}[\gamma E(t)-(\varphi+\delta+d) I(t)], \\
& \frac{s^{\alpha}}{W^{\alpha}} \mathbb{N}^{+}[R(t)]-\sum_{k=0}^{n-1} \frac{s^{\alpha-(k+1)}}{\omega^{\alpha-k}}\left[D^{k} R\right]_{t=0}=\mathbb{N}^{+}[\omega Q(t)+\varphi I(t)-(\varepsilon+d) R(t),], \\
& \frac{s^{\alpha}}{W^{\alpha}} \mathbb{N}^{+}[V(t)]-\sum_{k=0}^{n-1} \frac{s^{\alpha-(k+1)}}{\omega^{\alpha-k}}\left[D^{k} V\right]_{t=0}=\mathbb{N}^{+}[\mu S(t)-(\theta+d) V(t)] .
\end{aligned}
$$

By the above equation, we get

$$
\begin{aligned}
& \mathbb{N}^{+}[S(t)]=\frac{1}{s}\left[S_{0}(t)\right]+\frac{\omega^{\alpha}}{s^{\alpha}} \mathbb{N}^{+}\left[\rho Q(t)+\theta V(t)+\varepsilon R(t)-\left(\beta \sigma \pi r_{0}^{2} I(t)+\mu+\right.\right. \\
& d) S(t)], \\
& \mathbb{N}^{+}[E(t)]=\frac{1}{S}\left[E_{0}(t)\right]+\frac{\omega^{\alpha}}{s^{\alpha}} \mathbb{N}^{+}\left[\beta \sigma \pi r_{0}^{2} S(t) I(t)-(\gamma+d) E(t)\right], \\
& \mathbb{N}^{+}[I(t)]=\frac{1}{S}\left[I_{0}(t)\right]+\frac{\omega^{\alpha}}{s^{\alpha}} \mathbb{N}^{+}[\gamma E(t)-(\varphi+\delta+d) I(t)], \\
& \mathbb{N}^{+}[R(t)]=\frac{1}{S}\left[R_{0}(t)\right]+\frac{\omega^{\alpha}}{s^{\alpha}} \mathbb{N}^{+}[\omega Q(t)+\varphi I(t)-(\varepsilon+d) R(t),], \\
& \mathbb{N}^{+}[V(t)]=\frac{1}{S}\left[V_{0}(t)\right]+\frac{\omega^{\alpha}}{s^{\alpha}} \mathbb{N}^{+}[\mu S(t)-(\theta+d) V(t)] .
\end{aligned}
$$

On employing inverse $N T$ on Eq. (20), we have

$$
\begin{aligned}
S(t)=S_{0}(t)+\mathbb{N}^{-1}\left[\frac{\omega^{\alpha}}{s^{\alpha}} \mathbb{N}^{+}[\rho Q(t)+\theta V(t)+\varepsilon R(t)\right. \\
\left.\left.-\left(\beta \sigma \pi r_{0}^{2} I(t)+\mu+d\right) S(t)\right]\right] \\
E(t)=E_{0}(t)+\mathbb{N}^{-1}\left[\frac{\omega^{\alpha}}{s^{\alpha}} \mathbb{N}^{+}\left[\beta \sigma \pi r_{0}^{2} S(t) I(t)-(\gamma+d) E(t)\right]\right] \\
I(t)=I_{0}(t)+\mathbb{N}^{-1}\left[\frac{\omega^{\alpha}}{s^{\alpha}} \mathbb{N}^{+}[\gamma E(t)-(\varphi+\delta+d) I(t)]\right] \\
R(t)=R_{0}(t)+\mathbb{N}^{-1}\left[\frac{\omega^{\alpha}}{s^{\alpha}} \mathbb{N}^{+}[\omega Q(t)+\varphi I(t)-(\varepsilon+d) R(t),]\right] \\
V(t)=V_{0}(t)+\mathbb{N}^{-1}\left[\frac{\omega^{\alpha}}{s^{\alpha}} \mathbb{N}^{+}[\mu S(t)-(\theta+d) V(t)]\right]
\end{aligned}
$$

Let us consider that, the series solution for $S(t), E(t), I(t), R(t)$ and $V(t)$ respectively as follows 


$$
\begin{aligned}
S(t) & =\sum_{n=0}^{\infty} S_{n}(t), & E(t)=\sum_{n=0}^{\infty} E_{n}(t), \\
I(t) & =\sum_{\substack{n=0 \\
\infty}}^{\infty} I_{n}(t), & R(t)=\sum_{n=0}^{\infty} R_{n}(t), \\
V(t) & =\sum_{n=0}^{\infty} V_{n}(t) . &
\end{aligned}
$$

Note that, $S I=\sum_{n=0}^{\infty} P_{n}$ is represents the nonlinear terms and they are known as the Adomian polynomials. By the help of these terms, then Eq. (21) becomes

$$
\begin{aligned}
& \sum_{n=0}^{\infty} S_{n}(t)=S_{0}(t) \\
& +\mathbb{N}^{-1}\left[\frac { \omega ^ { \alpha } } { s ^ { \alpha } } \mathbb { N } ^ { + } \left[\rho Q(t)+\theta \sum_{n=0}^{\infty} V_{n}(t)+\varepsilon \sum_{n=0}^{\infty} R_{n}(t)\right.\right. \\
& \left.-\left(\beta \sigma \pi r_{0}^{2} \sum_{n=0}^{\infty} P_{n}+\mu \sum_{n=0}^{\infty} S_{n}(t)+d \sum_{n=0}^{\infty} S_{n}(t)\right)\right] \text {, } \\
& \sum_{n=0}^{\infty} E_{n}(t)=E_{0}(t)+\mathbb{N}^{-1}\left[\frac{\omega^{\alpha}}{s^{\alpha}} \mathbb{N}^{+}\left[\beta \sigma \pi r_{0}^{2} \sum_{n=0}^{\infty} P_{n}-(\gamma+d) \sum_{n=0}^{\infty} E_{n}(t)\right]\right] \text {, } \\
& \sum_{n=0}^{\infty} I_{n}(t)=I_{0}(t)+\mathbb{N}^{-1}\left[\frac{\omega^{\alpha}}{s^{\alpha}} \mathbb{N}^{+}\left[\gamma \sum_{n=0}^{\infty} E_{n}(t)-(\varphi+\delta+d) \sum_{n=0}^{\infty} I_{n}(t)\right]\right], \\
& \sum_{n=0}^{\infty} R_{n}(t)=R_{0}(t)+\mathbb{N}^{-1}\left[\frac { \omega ^ { \alpha } } { s ^ { \alpha } } \mathbb { N } ^ { + } \left[\omega Q(t)+\varphi \sum_{n=0}^{\infty} I_{n}(t)-(\varepsilon+\right.\right. \\
& \text { d) } \left.\left.\sum_{n=0}^{\infty} R_{n}(t)\right]\right] \text {, } \\
& \sum_{n=0}^{\infty} V_{n}(t)=V_{0}(t)+\mathbb{N}^{-1}\left[\frac{\omega^{\alpha}}{s^{\alpha}} \mathbb{N}^{+}\left[\mu \sum_{n=0}^{\infty} S_{n}(t)-(\theta+d) \sum_{n=0}^{\infty} V_{n}(t)\right]\right] \text {. }
\end{aligned}
$$

By the assist of the above system with prescribed initial conditions, we can find the terms of the series solution for the projected model systematically. Then, we establish the series solutions as

$$
\begin{aligned}
& S(t)=\sum_{n=0}^{\infty} S_{n}(t)=S_{0}(t)+S_{1}(t)+S_{2}(t)+\cdots \\
& E(t)=\sum_{\substack{n=0 \\
\infty}}^{\infty} E_{n}(t)=E_{0}(t)+E_{1}(t)+E_{2}(t)+\cdots \\
& I(t)=\sum_{n=0}^{\infty} I_{n}(t)=I_{0}(t)+I_{1}(t)+I_{2}(t)+\cdots
\end{aligned}
$$




$$
\begin{aligned}
& R(t)=\sum_{n=0}^{\infty} R_{n}(t)=R_{0}(t)+R_{1}(t)+R_{2}(t)+\cdots \\
& V(t)=\sum_{n=0}^{\infty} V_{n}(t)=V_{0}(t)+V_{1}(t)+V_{2}(t)+\cdots
\end{aligned}
$$

\section{Numerical results and discussion}

The investigation and analysis of mathematical models representing the network access control is a hot topic in this era. More importantly, using the efficient solution procedure and nonlocal operator, it attracted many scholars. In this work, we find the solution for a system of nonlinear equations exemplifying the projected model and further captured its corresponding consequences in the plots to confirm the reliability of the method employed and operator incorporated. With the assist of Figure 1, the differential system has been presented with specified parameters and corresponding values of the parameters are cited in Table 1.

Table 1. The specific value of the parameters presented in the projected system [38].

\begin{tabular}{|c|c|}
\hline Parameters & Values \\
\hline$\rho$ & 0.3 \\
\hline$\theta$ & 0.06 \\
\hline$\varepsilon$ & 0.3 \\
\hline$\beta$ & 0.1 \\
\hline$\sigma$ & 0.3 \\
\hline$\mu$ & 0.3 \\
\hline$r_{0}$ & 1 \\
\hline$d$ & 0.003 \\
\hline$\gamma$ & 0.25 \\
\hline$\lambda$ & 0.33 \\
\hline$\varphi$ & 0.4 \\
\hline$\delta$ & 0.07 \\
\hline$\omega$ & 0.01 \\
\hline
\end{tabular}

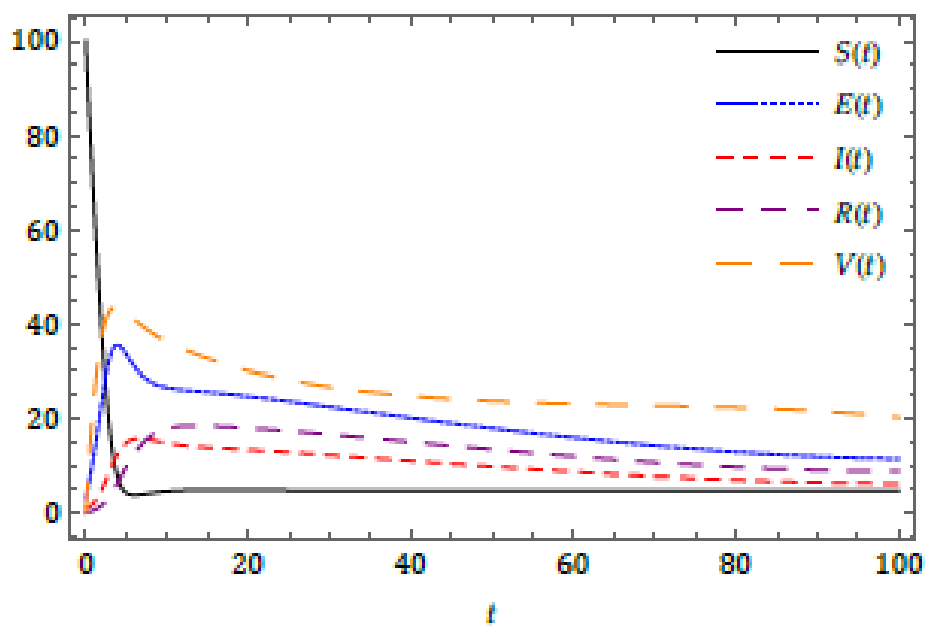

Figure 2. Nature of projected model for classical order using Table 1. 
The response for the classical order model has be captured in Figure 2 using the initial conditions

$\mathcal{Q}_{0}=5, \quad S(0)=S_{0}(t)=100, \quad E(0)=E_{0}(t)=3, \quad I(0)=I_{0}(t)=1$, $R(0)=R_{0}(t)=0, \quad V(0)=V_{0}(t)=0$.

Further, the nature of the result obtained by FNDM is presented in Figure 3 using the values of the parameters defined in Table 1. Form the Figures 2 and 3; we can see that the degree of freedom offered by the considered fractional operator related to classical model and also plots show some simulating results and consequences associated to hereditary properties and history-based results. Moreover, the current research can help to study a wide range of technological models exemplifying essential for the development of science and technology.

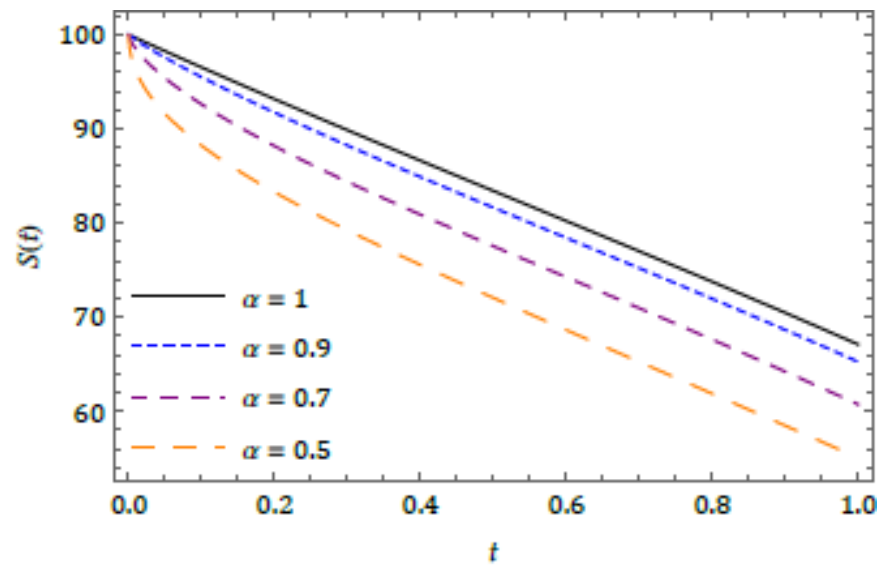

(a)

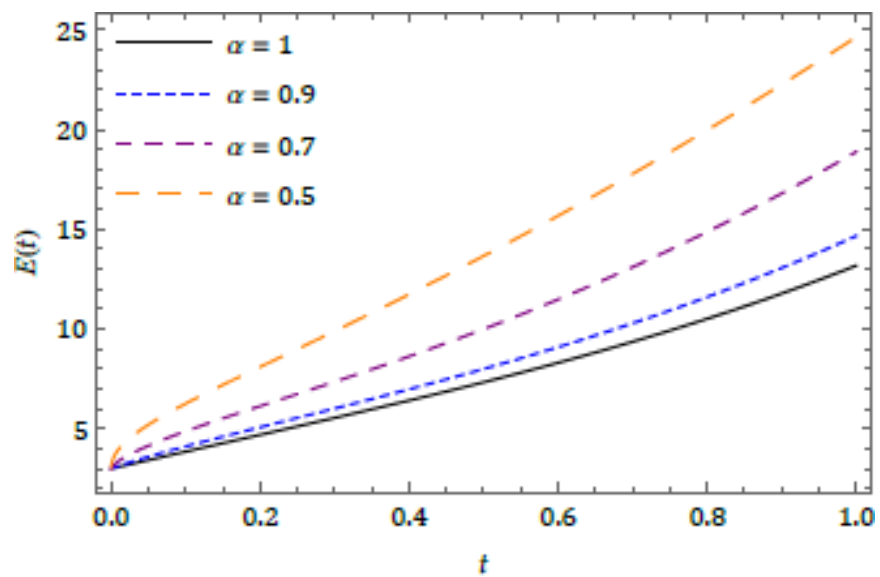

(b)

Figure 3. Nature of the obtained solution for $(a)$ susceptible $(S),(b)$ exposed $(E),(c)$ infectious $(I),(d)$ recovered $(R)$ and $(e)$ vaccinated $(V)$, with distinct $\alpha$ by the help of Table 1. 


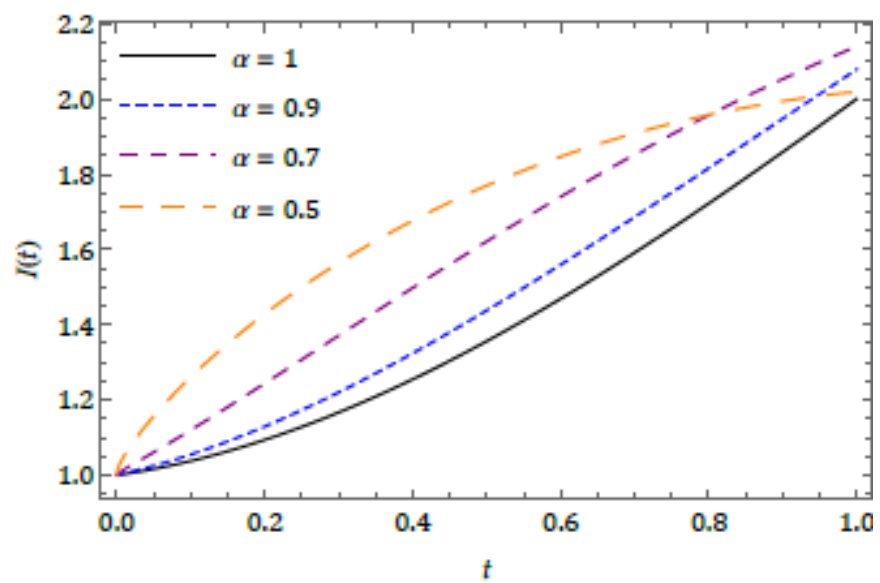

(c)

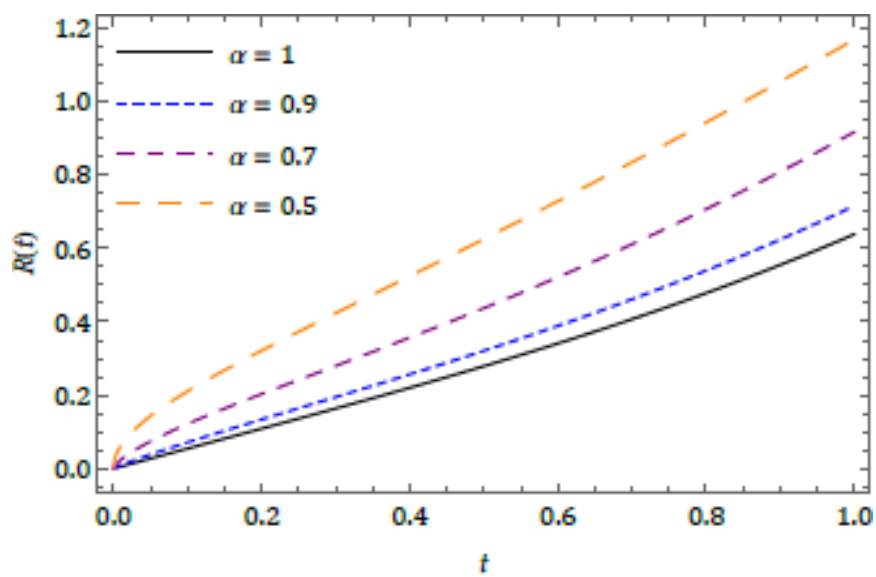

(d)

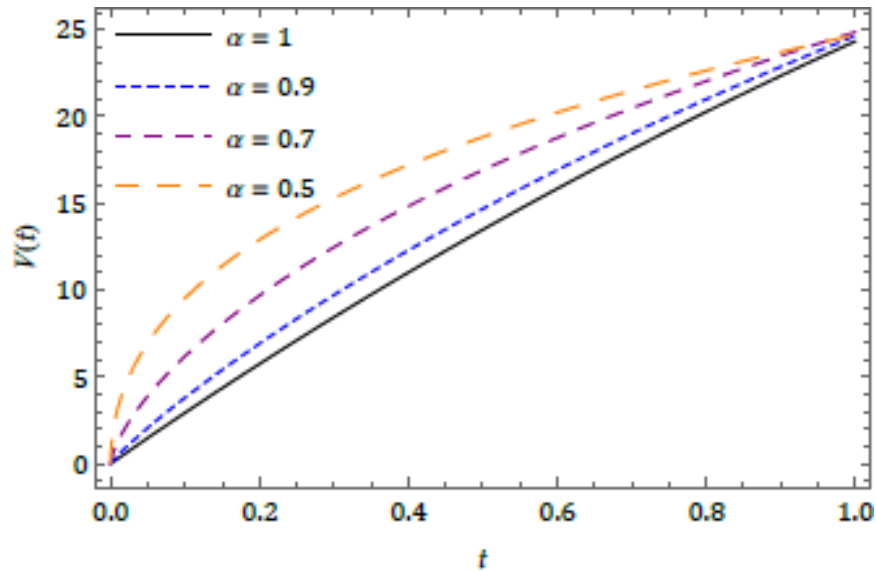

$(\boldsymbol{e})$

Figure 3 (Continued). Nature of the obtained solution for $(a)$ susceptible $(S),(b)$ exposed $(E),(c)$ infectious $(I),(d)$ recovered $(R)$ and $(e)$ vaccinated $(V)$, with distinct $\alpha$ by the help of Table 1 .

\section{Conclusion}

The fractional-order system exemplifying the network access control in the frame of the mathematical model is lucratively analysed and find the corresponding solution using efficient scheme (namely, FNDM) and considered nonlinear system exemplify 
computer networking solution that uses a set of protocols to implement and define. The obtained results by the considered solution procedure are more interesting associated with those is accessible in the literature. The considered method evaluates the solution for the complex nonlinear coupled systems without hiring any conversion, perturbation or discretization. The plots authorize the reliability of the hired algorithm and the effect of fractional order while we analysing biological models. Related to significances available in the literature, the attained results with the projected scheme are more stimulating and interesting. This study confirms, the phenomena conspicuously are contingent on the time history and the time instant, and these can be proficiently exemplified by the help of the fundamental notion of FC. The investigations of these types of models can provide a new path to analyse more real-world problems and it opens the door for employing an efficient method to study nonlinear phenomena associated with science and technology.

\section{References}

[1] Caputo, M., Elasticita e Dissipazione, Zanichelli, Bologna, (1969).

[2] Miller, K. S. and Ross, B., An introduction to fractional calculus and fractional differential equations, A Wiley, New York, (1993).

[3] Podlubny, I., Fractional Differential Equations, Academic Press, New York, (1999).

[4] Kilbas, A. A., Srivastava, H. M. and Trujillo, J. J., Theory and applications of fractional differential equations, Elsevier, Amsterdam, (2006).

[5] Baleanu, D., Guvenc, Z. B. and Machado, T. J. A., New trends in nanotechnology and fractional calculus applications, Springer Dordrecht Heidelberg, London New York, (2010).

[6] Prakasha, D. G. and Veeresha, P., Analysis of Lakes pollution model with Mittag-Leffler kernel, J. Ocean Eng. Sci., 5 (4), 310-322, (2020).

[7] Baleanu, D., Wu, G. C. and Zeng, S. D., Chaos analysis and asymptotic stability of generalized Caputo fractional differential equations, Chaos Solitons Fractals, 102, 99-105, (2017).

[8] Veeresha, P., Prakasha, D. G. and Baskonus, H. M., New numerical surfaces to the mathematical model of cancer chemotherapy effect in Caputo fractional derivatives, Chaos, 29, (013119), (2019).

[9] Baskonus, H. M., Sulaiman, T. A. and Bulut, H., On the new wave behavior to the Klein-Gordon-Zakharov equations in plasma physics, Indian J. Phys., 93, (3), 393-399, (2019).

[10] Veeresha, P. and Prakasha, D. G., Solution for fractional generalized Zakharov equations with Mittag-Leffler function, Results Eng., 5, 1-12, (2020).

[11] Prakasha, D. G., Malagi, N. S. and Veeresha, P., New approach for fractional Schrödinger-Boussinesq equations with Mittag-Leffler kernel, Math. Meth. Appl. Sci., (2020).

[12] Gao, W., Baskonus, H. M. and Shi, L., New investigation of Bats-HostsReservoir-People coronavirus model and apply to 2019-nCoV system, Adv. Differ. Equ., 391, (2020).

[13] Cattani, C. and Pierro, G., On the fractal geometry of DNA by the binary image analysis, Bull. Math. Biol., 75, (9), 1544-1570, (2013). 
[14] Gao, W., Veeresha, P., Prakasha, D. G. and Baskonus, H.M., Novel dynamical structures of 2019-nCoV with nonlocal operator via powerful computational technique, Biology, 9, (5), (2020).

[15] Gao, W., Veeresha, P., Baskonus, H. M., Prakasha, D. G. and Kumar, P., A new study of unreported cases of 2019-nCOV epidemic outbreaks, Chaos Solitons Fractals, 138, (2020).

[16] Cattani, C., Haar wavelet-based technique for sharp jumps classification, Math. Comput. Model., 39, (2-3), 255-278, (2004).

[17] Gao, W., Yel, G., Baskonus, H.M. and Cattani, C., Complex solitons in the conformable (2+1)-dimensional Ablowitz-Kaup-Newell-Segur equation, AIMS Math., 5, (1), 507-521, (2020).

[18] Al-Ghafri, K. S. and Rezazadeh, H., Solitons and other solutions of (3+1)dimensional space-time fractional modified KdV-Zakharov-Kuznetsov equation, Appl. Math. Nonlinear Sci., 4, (2), 289-304, (2019).

[19] Dananea, J., Allalia, K. and Hammouch, Z., Mathematical analysis of a fractional differential model of HBV infection with antibody immune response, Chaos Solitons Fractals, 136, 109787, (2020).

[20] Singh, J., Kumar, D., Hammouch, Z. and Atangana, A., A fractional epidemiological model for computer viruses pertaining to a new fractional derivative, Appl. Math. Comput., 316, 504-515, (2018).

[21] Ravichandran, C., Logeswari, K. and Jarad, F., New results on existence in the framework of Atangana-Baleanu derivative for fractional integro-differential equations, Chaos Solitons Fractals, 125, 194-200, (2019).

[22] Veeresha, P., Prakasha, D. G. and Baskonus, H. M., An efficient technique for coupled fractional Whitham-Broer-Kaup equations describing the propagation of shallow water waves, Advances in Intelligent Systems and Computing, 49-75, (2020).

[23] Kiran, M. S., et al., A mathematical analysis of ongoing outbreak COVID-19 in India through nonsingular derivative, Numer. Meth. Partial Differ. Equ., (2020), DOI: 10.1002/num.22579.

[24] Veeresha, P., Prakasha, D. G., Singh, J., Kumar, D. and Baleanu, D., Fractional Klein-Gordon-Schrödinger equations with Mittag-Leffler memory, Chinese J. Phy., 68, 65-78, (2020).

[25] Subashini, R., Ravichandran, C., Jothimani, K. and Baskonus, H. M., Existence results of Hilfer integro-differential equations with fractional order, Discrete Contin. Dyn. Syst. Ser. S, 13, (3), 911-923, (2020).

[26] Veeresha, P. and Prakasha, D. G. Novel approach for modified forms of Camassa-Holm and Degasperis-Procesi equations using fractional operator, Commun. Theor. Phys., 72, (10) (2020),

[27] Panda, S. K., Abdeljawad, T. and Ravichandran, C., Novel fixed point approach to Atangana-Baleanu fractional and Lp-Fredholm integral equations, Alexandria Eng. J., 59, (4), 1959-1970, (2020).

[28] Veeresha, P., Prakasha, D. G. and Hammouch, Z., An efficient approach for the model of thrombin receptor activation mechanism with Mittag-Leffler function, Nonlinear Analysis: Problems, Applications and Computational Methods, 44-60, (2020).

[29] Subashini, R., Jothimani, K., Nisar, K. S. and Ravichandran, C. New results on nonlocal functional integro-differential equations via Hilfer fractional derivative, Alexandria Eng. J., 59, (5), 2891-2899, (2020). 
[30] Alqudah, M. A., Ravichandran, C., Abdeljawad, T. and Valliammal, N., New results on Caputo fractional-order neutral differential inclusions without compactness, Adv. Differ. Equ., 528, (2019).

[31] Veeresha, P., Prakasha, D. G. and Baleanu, D., Analysis of fractional SwiftHohenberg equation using a novel computational technique, Math. Meth. Appl. Sci., 43, (4), 1970-1987, (2020).

[32] Logeswari, K. and Ravichandran, C., A new exploration on existence of fractional neutral integro-differential equations in the concept of AtanganaBaleanu derivative, Phys. A, 544, (2020), DOI: 10.1016/j.physa.2019.123454

[33] Veeresha, P., Prakasha, D. G. and Kumar, D., Fractional SIR epidemic model of childhood disease with Mittag-Leffler memory, Fractional Calculus in Medical and Health Science, 229-248, (2020).

[34] Akyildiz, I. F., Su, W., Sankarasubramaniam, Y. and Cayirci, E., Wireless sensor networks: A survey, Computer Networks, 38, (4), 393-422, (2002).

[35] Nwokoye, C. H., Umeh, I., Nwanze, M. and Alao, B. F., Analyzing time delay and sensor distribution in sensor networks, IEEE African Journal of Computing \& ICT 8, 1, 159-164, (2015).

[36] Giannetsos, T., Dimitriou, T. and Prasad, N. R., Self-propagating worms in wireless sensor networks, ACM CoNEXT-Student Workshop'09, 31-32, (2009).

[37] Gupta, A. and Gupta, A. K., A survey: Detection and prevention of wormhole attack in wireless sensor networks, Global Journal of Computer Science and Technology: E Network, Web \& Security, 14, 23-31, (2014).

[38] ChukwuNonso, H. N., Mbeledogu, N., Umeh, I. I., Ihekeremma, and Ejimofor, A., Modeling the effect of network access control and sensor random distribution on worm propagation, I.J. Modern Education and Computer Science, 11, 49-57, (2017).

[39] Adomian, G., A new approach to nonlinear partial differential equations, J. Math. Anal. Appl., 102, 420-434, (1984).

[40] Khan, Z. H. and Khan, W. A., N-Transform-properties and applications, NUST J. Engg. Sci., 1, (1), 127-133, (2008).

[41] Rawashdeh, M. S., The fractional natural decomposition method: theories and applications, Math. Meth. Appl. Sci., 40, 2362-2376, (2017).

[42] Rawashdeh, M. S. and Maitama, S., Finding exact solutions of nonlinear PDEs using the natural decomposition method, Math. Meth. Appl. Sci., 40, 223-236, (2017).

[43] Prakasha, D. G., Veeresha, P. and Rawashdeh, M. S., Numerical solution for $(2+1)$-dimensional time-fractional coupled Burger equations using fractional natural decomposition method, Math. Meth. Appl. Sci., 42, (10), 3409-3427, (2019).

[44] Veeresha, P., Prakasha, D.G. and Singh, J., Solution for fractional forced KdV equation using fractional natural decomposition method, AIMS Math., 5, (2), 798-810, (2019).

[45] Rawashdeh, M. S., Solving fractional ordinary differential equations using FNDM, Thai Journal of Mathematics, 17, (1), 239-251, (2019).

[46] Prakasha, D. G., Veeresha, P. and Baskonus, H. M., Two novel computational techniques for fractional Gardner and Cahn-Hilliard equations, Comp. Math. Methods, 1, (2), 1-19, (2019). 
[47] Veeresha, P. and Prakasha, D. G. An efficient technique for two-dimensional fractional order biological population model, Int. J. Mod. Simul. Sci. Comput., (2050005), 1-17, (2020).

[48] Mishra, B. K. and Keshri, N., Mathematical model on the transmission of worms in wireless sensor network, Appl. Math. Model., 37, 4103-4111, (2013).

[49] Chunbo, L. and Chunfu, J., Modeling passive propagation of malwares on the WWW, Physics Procedia, 33, 271-278, (2012).

[50] Yao, Y., Guo, L., Guo, H., Yu, G., Gao, F. X. and Tong, X. J., Pulse quarantine strategy of internet worm propagation: Modeling and analysis, Computers and Electrical Engineering, 38, 1047-1061, (2012).

[51] Khayam, S. A. and Radha, H., Using signal processing techniques to model worm propagation over wireless sensor networks, IEEE Signal Processing Magazine, 164-169, (2006).

[52] Wang, X. and Li, Y., An improved SIR model for analyzing the dynamics of worm propagation in wireless sensor networks, Chinese Journal of Electronics, 18, 8-12, (2009).

[53] Wang, X., Li, Q. and Li, Y., EiSIRS: A formal model to analyze the dynamics of worm propagation in wireless sensor networks, J. Comb. Optim., 20, 47-62, (2010).

[54] Wang, Y. and Yang, X., Virus spreading in wireless sensor networks with a medium access control mechanism, Chinese Phy. B, 22, 40200-40206, (2013).

[55] Zhang, Z. and Si, F., Dynamics of a delayed SEIRS-V model on the transmission of worms in a wireless sensor network, Adv. Diff. Equ., 295, (2014).

[56] Mittag-Leffler, G. M., Sur la nouvelle fonction $E_{\alpha}(x)$, C. R. Acad. Sci. Paris, 137, 554-558, (1903).

[57] Loonker, D. and Banerji, P. K., Solution of fractional ordinary differential equations by natural transform, Int. J. Math. Eng. Sci., 12, (2), 1-7, (2013). 